\title{
DYNAMIC SPECTRUM DETECTION Via COMPRESSIVE SENSING
}

\author{
Olusegun Odejide $^{1}$, Michael Odeyomi ${ }^{2}$ and Annamalai Annamalai ${ }^{1}$ \\ ${ }^{1}$ Department of Electrical \& Computer Engineering, Prairie View A\&M University, \\ Prairie View, TX USA \\ femiodejide@yahoo.com, aaannamalai@pvamu.edu \\ ${ }^{2}$ Shell Oil Company, Houston, TX USA \\ michael.odeyomi@shell.com
}

\begin{abstract}
Spectrum congestion is a major concern in both military and commercial wireless networks. To support growing demand for ubiquitous spectrum usage, Cognitive Radio is a new paradigm in wireless communication that can be used to exploit unused part of the spectrum by dynamically adjusting its operating parameters. While cognitive radio technology is a promising solution to the spectral congestion problem, efficient methods for detecting white spaces in wideband radio spectrum remain a challenge. Conventional methods of detection are forced to use the high sampling rate requirement of Nyquist criterion. In this paper, the feasibility and efficacy of using compressive sensing (CS) algorithms in conjunction with Haar wavelet for identifying spectrum holes in the wideband spectrum is explored. Compressive sensing is an emerging theory that shows that it's possible to achieve good reconstruction, at sampling rates lower than that specified by Nyquist. CS approach is robust in AWGN and fading channel.
\end{abstract}

\section{KEYWORDS}

Compressive Sensing, Nyquist rate, Fading channel

\section{INTRODUCTION}

Wireless spectrum is in scarcity but recent research identified that there exists low spectrum utilization over wide frequency ranges including licensed spectrum bands. To utilize the spectrum more efficiently, spectrum sensing for underutilized bands are gaining momentum currently. The key motivation for this work is to detect the underutilized spectrum or spectrum holes efficiently and dynamically reuse the holes for the cognitive radio (CR) network application. Cognitive Radio is a single or network nodes that can sense and detect changes in the environment to operate in the unused spectrum to create faster, cheaper, scalable and robust multimedia wireless communications. Cognitive radio, a software defined intelligent radio system is in the heart of opportunistic wireless communication where the system employs spectrum sensing mechanism and utilize the spectrum hole for opportunistic communications without interfering with the primary user's communications. Open spectrum sensing for the cognitive radio (CR) network faces considerable technical challenges. One of the key challenges for CR network is the spectrum sensing.

Dynamic access of wideband spectrum requires fast and accurate sensing of used and unused frequency band. In cognitive radio (CR) networks where dynamic utilization of available spectrum is implemented, unlicensed CR users constantly search for free/unoccupied frequency band. These frequency band that are unoccupied by communication systems holding the license of the said spectrum, are opportunistically used for transmission by the CRs. The FCC recently on November 14, 2008, released its second report and memorandum of opinion and order, which is commonly referred to as white spaces decision [1]. The rule governs the operation of DOI : $10.5121 /$ ijcnc.2012.4207 
non-broadcast devices in the TV broadcast band. By so doing, this rule is expected to lead to significant benefits for both businesses and consumers by promoting more efficient and effective use of the available analog TV spectrum. This ruling will further bring to forefront the idea of dynamic spectrum access. The foremost task towards achieving the ruling is sensing and identification of unused holes in the spectrum.

Transmitter detection and interference-based detection [2] are the two broad categories for frequency spectrum 'holes' detection methods. Transmitter detection method includes matched filter detection [3], energy detection [4,5], cyclostationary feature detection [6] and wavelet detection [7]. Matched filter detection approach is a coherent detector, it is optimal in stationary Gaussian noise, and achieves high gain in less time. Its drawback lies in that apriori information (such as modulation type and order, pulse shape, etc) about the signal being detected is required. In a situation whereby apriori information about the signal is not known, the energy detector method is highly useful as long as noise power is known. The method's susceptibility to noise power uncertainty, poor performance under low SNR conditions and inability to detect signal types are among its shortcomings.

Wavelet detection makes use of the multiresolution analysis mechanism of wavelet transform. An input signal is decomposed into different frequency components, and the resolution of each component is matched to its scales. Wavelet can be very useful for wideband channel signal detection; it offers a flexible detection capability as opposed to the conventional use of multiple narrowband bandpass filters [8]. One major disadvantage of using wavelet techniques for spectrum sensing is that a high sampling rate is often required in practice for large bandwidth characterization [9]. The cyclostationarity feature detection method is robust against noise; but, it is computationally intensive and requires long time.

While cognitive radio technology is a promising solution to the spectral congestion problem, efficient methods for detecting the white spaces or spectral holes in wideband radio spectrum remain a challenge. Conventional methods of detection are forced to use the high sampling rate requirement of Nyquist criterion. Moreover, timing requirements limit the number of samples that can be taken from the composite signals. In this paper, the feasibility and efficacy of using compressive sensing (CS) algorithms in conjunction with Haar wavelet for identifying spectrum holes in the wideband spectrum is explored. One major problem with wideband spectrum sensing is the high cost of current analog-to-digital hardware technology required for data acquisition based on Nyquist criteria specification at such high frequency. In contrast with the above mentioned strategies for spectrum sensing, Compressive sensing has the potential of achieving frequency spectrum sensing with fewer number of samples with different channel conditions without a priori information. The effects of additive and multiplicative noise, fading distributions, sample size, and CS reconstruction algorithms are investigated.

The CS approach proposed in this work enables a simple cognitive radio receiver implementation for wideband spectrum sensing by considerably reducing the number of samples required for power spectral density reconstruction. It has been demonstrated via simulation that the CS approach is also robust in the presence of additive white Gaussian channel and multiplicative noise (Rayleigh and Ricean multipath fading channels). The effects of fade distributions and the sample size on CS reconstruction algorithms were also investigated.

\section{Compressive Sensing And Problem Statement}

\subsection{Compressive Sensing}

Nyquist theory defined the required sampling rate for effective reconstruction of signals to be at least twice the maximum bandwidth or frequency component of the signal. For signals in broadband communication ranges, existing hardware limitation makes it difficult or impossible 
to achieve Nyquist rate in circuitry. The RF signal acquisition cost based on the currently available analog-to-digital hardware technology is very high. In addition to the condition specified by Nyquist criteria, dynamically changing spectrum needs to be monitored frequently; however because of the large bandwidth, only a limited number of measurements can be taken.

This condition hinders the ability to carry out good signal reconstruction since samples cannot be taken at the rate specified by Nyquist. Another major problem is that wireless fading often affects adequate spectrum detection mechanism. If adequate and proper detection of occupied bandwidth is not achieved, this may lead to a situation whereby the cognitive radio transmit over a channel that is occupied by an active user, which will mostly result in unwanted interference.

Recent research results in the area of compressive sampling or compressed sensing have shown that it is possible to reconstruct a signal with far fewer measurements than the traditional Nyquist criteria specified, as long as the signal is sparse (or nearly sparse) in some basis or frame [10]-[11]. According to CS theories, a vector of discrete-time signal $\mathrm{x}$ is $\mathrm{K}$-sparse in some basis matrix $\Psi$, i.e.,

$x=\Psi a$

where $a$ is an $\mathrm{N}$ by 1 vector and has only $\mathrm{K}$ non-zero elements and $(\mathrm{N}-\mathrm{K})$ zero elements. $\Psi$ is a $\mathrm{N}$ by $\mathrm{N}$ matrix. $x$ is compressible if a has just a few large coefficients and many small coefficients [11]. $\mathrm{d}=\mathrm{K} / \mathrm{N}$ is defined as the sparsity of $\mathrm{x}$. We can reconstruct this signal successfully with high probability from L measurements, where L depends on reconstruction algorithms and usually $\mathrm{L}<<\mathrm{N}$. One key requirement of this theory is that these measurements should be sparse or compressible in some basis. Although the measurement process may be performed independent of signal type, reconstruction algorithms typically require a priori information or assumptions in order to select an optimal or near optimal reconstruction basis.

The reconstruction can be performed using a variety of basis functions, frames, or dictionaries, including Fast Fourier Transform (FFT), Discrete Cosine Transform (DCT), Discrete Wavelet Transform (DWT), curvelets, etc. Wavelet transform has been proven to perform well for various applications such as in signal detection [12]. A good CS signal reconstruction depends on the sparseness or compressibility of the signal, the choice of reconstruction algorithm as well as the basis function. Due to low percentage of spectrum occupancy by active radios, the wireless signals in open-spectrum network are typically sparse in the frequency domain. Hence, the idea of CS signal reconstruction can be used to detect occupied spectral bands as illustrated in [13]-[14].

The unique feature of compressive sensing based spectrum detection where few samples at subNyquist rate is required in order to detect holes in the spectrum has increased interest in this research area. In [15], the author looked into ways of optimizing the sparsifying based in order to improve the spectrum sensing efficiency. Also S Hong [16] demonstrated how utilizing a Bayesian Compressive Sensing framework in spectrum sensing can result into significantly less computational complexity. Our goal in this paper is to show the efficacy of the CS based spectrum sensing technique using different reconstruction algorithm in diverse multipath fading environment.

\subsection{Reconstruction Algorithm}

One of the factors that determine the accuracy of the CS signal reconstruction is the algorithm. Orthogonal Matching Pursuit (OMP), Basis Pursuit (BP), Tree Orthogonal Matching Pursuit (TOMP), Stepwise Orthogonal Matching Pursuit (STOMP), Gradient Projection ), and a row action method using only vector arithmetic are among the several algorithms that has been proposed for solving the $l_{1}$ minimization problem. The approaches vary in their speed, ability to handle large data sets, and accuracy under various conditions. The choice of basis function for 
projecting the signal also determines the accuracy of the reconstructed signal. Brief but detailed explanations of the reconstruction algorithms used for this paper is given below:

A compressed sensing solver that seeks to compute the minimum $\ell_{0}$ norm solution is the Orthogonal Matching Pursuit (OMP) algorithm. This algorithm attempts to determine which columns of the pseudo random matrix $\Phi$ are most correlated to the measurement matrix y. For example, when the signal is reconstructed through orthogonal matching pursuit (OMP) algorithm, $\mathrm{L}$ is approximately $2 \mathrm{~K} \log (\mathrm{N})$ achieve a reasonable reconstruction quality. Consider a general linear measurement process that computes $\mathrm{L}$ inner products between $\mathrm{x}$ and a collection of vectors $\left\{\Phi_{\mathrm{j}}\right\} \mathrm{j}=1$ as in $\mathrm{y}=\left(\mathrm{x}, \Phi_{\mathrm{j}}\right)$. Arrange the measurement $\mathrm{y}_{\mathrm{j}}$ in a $\mathrm{L} \mathrm{x} 1$ vector $\mathrm{y}=\left[\mathrm{y}_{1}, \mathrm{y}_{2}, \ldots \ldots \ldots, \mathrm{y}_{\mathrm{L}}\right]^{\mathrm{T}}$ and the measurement vector $\Phi^{\mathrm{T}}$, as rows in a $\mathrm{L} \mathrm{x} \mathrm{N}$ matrix $\Phi$. Then , by substituting $\Psi$ from (1), y can be written as

$$
y=\Phi x=\Phi \Psi a=\theta a
$$

where $\theta=\Phi \Psi a$ is a $\mathrm{L}$ by $\mathrm{N}$ projection matrix.

Basis Pursuit is a convex relaxation that offers another approach to sparse approximation. The fundamental idea is that the number of terms in a representation can be approximated by the absolute sum of the coefficients. This absolute sum is a convex function, and so it can be minimized in polynomial time. Given an input signal s, the BP problem is

$$
\begin{aligned}
& \min _{\left\{b_{\omega}\right\}} \sum_{\omega \in \Omega}\left|b_{\omega}\right| \\
& \text { subject to } \sum_{\omega \in \Omega}\left|b_{\omega} \varphi_{\omega}=s\right|
\end{aligned}
$$

where $b_{\omega}$ is a collection of complex coefficients. One hopes that the nonzero coefficients in the solution of the BP problem will identify the atoms in the optimal representation of the input signal and their coefficients.

LASSO (Least Absolute Shrinkage and Selection Operator) is derivatives of LARS (Least Angle Regression) minimum $l_{l}$ norm reconstruction algorithm. This greedy algorithm take advantage of geometric correlation between the targeted and the coefficients most correlated with the measured response. LASSO algorithms take advantage of quadratic programming concepts because they produce a weighted minimum $l_{1}$ and $l_{2}$ norm solution.

\subsection{Problem Statement}

Assuming that a cognitive radio receiver receives a signal occupying $\mathrm{N}$ consecutive spectrum band with frequency boundaries located at $f_{0}<f_{l}<\ldots f_{n}$. The frequency response of the received signal can be categorized based on the level of the power spectral density of the received signal $r(t)$ and is illustrated in Fig. 1. If the PSD level is high, medium or low; the spectrum can be considered as black, grey or white. Whereby white hole and sometimes gray spaces can be opportunistically used by the CR for transmission, while the black hole are not to be used in order to avoid interferences.

In a wideband frequency spectrum situation, following Nyquist sampling criteria, very large samples will be required to effectively recover signal $r(t)$. The goal of each Cognitive radio is to effectively sense, classify and estimate the frequency spectrum of signal $r(t)$. Our focus is to be able to sense and classify (identify occupied frequency bands) the signal $r(t)$ under different channel conditions (noiseless, additive and multiplicative noise) as well as using different CS reconstruction algorithm. 


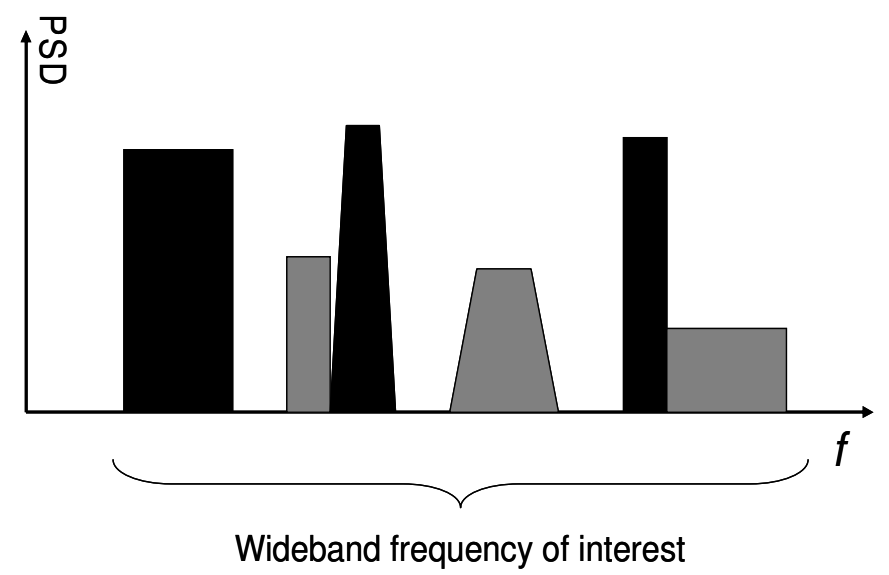

Figure 1. PSD of a wideband spectrum

In order to classify and estimate the frequency spectrum, we look at the power spectral density as the original signal, and then try to use CS based reconstruction algorithm to reconstruct the frequency spectrum provided it was under sampled. Samples required for spectrum reconstruction in a wideband frequency signal would have been difficult to implement, however using the theory of compressed sensing, since the PSD is sparse in nature, it is possible to detect the holes in the spectrum by reconstructing the PSD with fewer samples. It is also worth mentioning that since the original signal is the PSD of the wideband frequency spectrum, Haar wavelet happens to be the best sparsifying basis for reconstruction in compressive sensing. The efficacy of compressed sensing scheme in detecting holes in the spectrum is investigated, without noise and in the presence of noise (both multiplicative and additive).

\subsection{Multipath Fading Channel}

A principal feature of a cognitive radio (CR) is a reliable spectrum sensing technique which enables the CR to detect white spaces in the frequency band. This allows opportunistic access of the unlicensed (secondary) users to these unoccupied spaces without causing interference to licensed (primary) users. In many scenarios the CR may operate in a multipath fading environment where spectrum sensing must deal with the fading effects of the unknown primary PSD signal. It important to study the effects of multipath fading on the performance of Compressive Sensing (CS) based spectrum sensing algorithms.

The detection performance of a CR network system is critically affected by the multi-path characteristic of the environment. In a high speed wideband CR network, multi-path fading is one of the most serious transmission difficulties. However, there are also long term fluctuations in the characteristics of the wireless channel depending on the actual position of the Cognitive Radio and the mobile station, that is, in urban areas the signal is affected by multi-path propagation. Theorical results show that the channel capacity in a multi-path fading environment is always lower than that in a Gaussian noise environment. In a wideband frequency communication channel that has fading characteristics, it is very important to know what the degradations are in channel capacity due to multipath fading, and also to what degree the diversity schemes can bring the channel capacity up or down. The previous chapter centered on the effect of additive noise to CS based spectrum detection. This chapter is centered on invetigating the robustness of CS algorithms in spectrum sensing in the presence of additive and multiplicative noise.

The channel amplitude in a Rayleigh fading environment is always lower than that of a Gaussian noise environment. The fading channels are modeled as frequency non-selective slow Rayleigh and Rice fading channels corrupted by AWGN. Rayleigh and Ricean fading channels are useful models of real-world phenomena in wireless communications. These phenomena 
include multipath scattering effects, time dispersion, and Doppler shifts that arise from relative motion between the transmitter and receiver. The transmission path between a transmitter and receiver can vary from a simple line-of-sight (LOS) path to a non-line-of-sight (NLOS) path, which is a path that is severely obstructed by buildings, mountains, and foliage. Furthermore, when a CR moves in space, the speed of motion impacts how rapidly the signal level fades.

Two widely used models for fading channels are the Rayleigh fading channel and the Ricean fading channel, where the Rayleigh fading channel is actually a special case of the Ricean fading channel. The Rayleigh model is used when there is NLOS between transmitter and receiver, and all of the received signal power is due to multipath. The Ricean model is used when there is a LOS between transmitter and receiver, but a substantial portion of the received signal power is also due to multipath. When there is LOS between transmitter and receiver and virtually none of the received signal power is due to multi-path, the non-fading channel model is used.

Rayleigh fading is a type ofsmall-scale fading. If the multipath reflective paths are large in number and are all NLOS, the envelop of the received signal can be statistcally expressed by using a Rayleigh fading distribution. The Rayleigh fading distribution has a probability density function given by

$$
p(r)=\left\{\begin{array}{l}
\frac{r}{\sigma 2} \exp \left(-\frac{r^{2}}{2 \sigma^{2}}\right), r \geq 0 \\
0, \text { otherwise }
\end{array}\right.
$$

Where $r$ is the envelop amplitude of the received signal, and $\sigma^{2}$ is the time-average power of the multipath signal.

The Rice fading distribution has a probability density function given by

$$
p(r)=\left\{\begin{array}{cc}
\frac{r}{\sigma^{2}} \exp \left(-\frac{r^{2}+A^{2}}{2 \sigma^{2}}\right) I_{0}\left(\frac{A r}{\sigma^{2}}\right), & r \geq 0 \text { and } A \geq 0 \\
0 & r<0
\end{array}\right.
$$

Where $r$ is the envelope and $\sigma^{2}$ is average of the Rice fading signal. A is the peak amplitude of the dominant signal, and $I_{0}($.$) is the modified Bessel function of zero order, which is given by$

[17]

$$
I_{0}(z)=\sum_{n=0}^{\infty} \frac{z^{2 n}}{2^{2 n}(n !)^{2}}
$$

Note that the Rice fading distribution in equation (6) presents two extreme cases:

1. If the dominant signal $A=0, p(r)$ become the Rayleigh fading distribution in equation (5).

2. If the dominant signal $A$ is large, $p(r)$ becomes a Gaussian distribution.

The Rice fading distribution is usually expressed in terms of a parameter $K$ that is defined as the ratio between the deterministic signal power and the variance of the multipath. The parameter is given by

$k=\frac{A^{2}}{2 \sigma^{2}}$

Or in decibels

$$
K=20 \log \left(\frac{A}{\sqrt{2 \sigma}}\right)
$$


Where $k$ (or $\mathrm{K}$ ) is known as the Ricean factor that completely specifies the Ricean fading distribution. Note that the Ricean fading distribution becomes the Rayleigh fading distribution as $A \rightarrow 0, K \rightarrow-\infty d B$.

\subsection{Doppler Spread}

Due to the relative motion of transmitter and receiver in the CR networks, the Doppler shift is of great practical importance to take account of. The effect of the Doppler shift on the CR network receiver is prevalent. In a dense CR network the phenomenon of Doppler spread in which the spectrum of the transmitted signal is not displaced in the frequency by Doppler shift, but is actually spread out [18].

Doppler spread, Ds is a measure of spectral broadening, $f_{d}$ caused by the time rate of change of the wideband wireless channel. The Doppler spread is defined as the range of frequencies over the received Doppler power spectrum $S(v)$. The components range from $\left(f_{c}-\right.$ $\left.f_{d}\right)$ to $\left(f_{c}+f_{d}\right)$, where $f_{c}$ is the transmitted pure sinusoidal tone of frequency and $f_{d}$ is the Doppler shift.

$$
\begin{aligned}
& \text { The } S(\mathrm{v}) \text { at the } \mathrm{CR} \text { receiver is then given by } \\
& S(v)= \begin{cases}\frac{3}{2 \pi f_{m}}\left[1-\left(\frac{v-f_{s}}{f_{m}}\right)^{2}\right]^{-\frac{1}{2}},\left|f-f_{o}\right|<f_{m} \\
0, \quad \text { otherwise }\end{cases}
\end{aligned}
$$

Where $\mathrm{v}$ is the Doppler frequency shift and $f_{m}$ is the maximum Doppler shift given by

$$
f_{m}=\max \left(\left\|f_{d}\right\|\right)=\max \left(\frac{v}{\lambda}|\cos \theta|\right)=\frac{v}{\lambda}
$$

Where $f_{i}$ can be either positive or negative depending on the arrival angle $\theta$. V is the relative velocity, and $\lambda$ is the signal wavelength. Thus, in this case, the Doppler spread is obtained by

$$
D_{s}=f_{m}
$$

The knowledge of the Doppler power spectrum in equation 10 allows the determination of how much spectral broadening is imposed on the signal as a function of the change in the multipath channel state.

\section{NUMERICAL RESULTS}

In our simulations, based on the theory of compressive sensing, we considered a wideband of interest with the signal of interest being the frequency domain PSD of the spectrum. We employed the idea of CS signal reconstruction in order to detect occupied/unoccupied spectral bands. We decided to solve the problem using OMP, BP and LASSO reconstruction algorithm. These are three of several CS-based algorithms. Our simulations revealed that reconstruction of the signal is possible using any of these three algorithms.

The choice of how many measurements are required to accurately reconstruct the PSD of a wideband spectrum in noiseless environment is of topmost importance. A major implementation challenge of conventional spectral estimation methods lies in the very high sampling rates required, which have to operate at or above the Nyquist rate. Compressive Sensing is a method for acquisition of sparse signals at rates far lower than the Nyquist sampling rate. With CS the original PSD signal can be reconstructed using far fewer measurements than the Nyquist criterion. This theory helps to offer the much needed benefits for reduced transmission bandwidth as a result of compression ratio achievable.

Wideband PSD in frequency the domain are sparse and this is an advantage that allows the use of measurement matrix to sense the PSD and reconstruct the spectrum of the PSD by a 
$\mathrm{CS}$ reconstruction algorithm. Therefore, if the number of measurements is $\mathrm{L} \ll \mathrm{N}$, a high sampling rate is not required. Sub-Nyquist rate can be used to get a number of measurements.
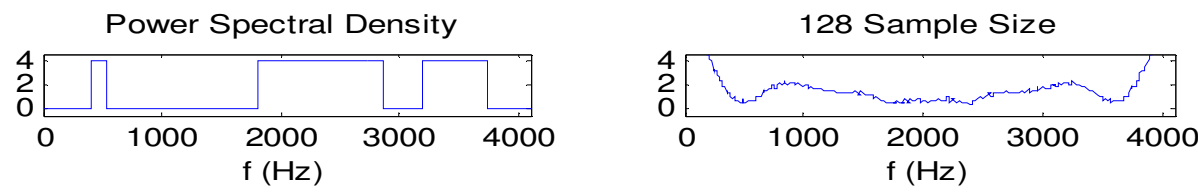

256 Sample Size

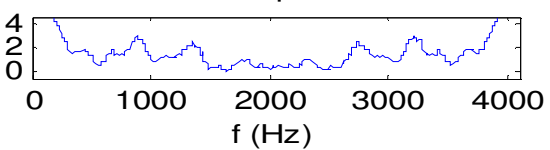

512 Sample Size

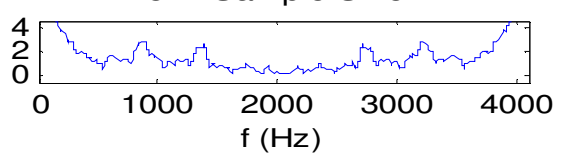

1024 Sample Size

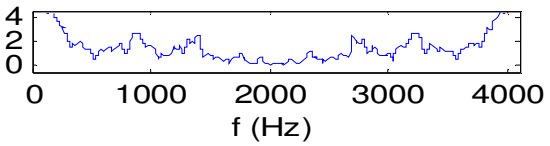

2048 Sample Size

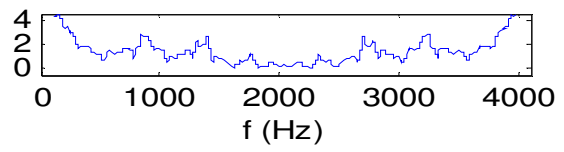

4096 Sample Size

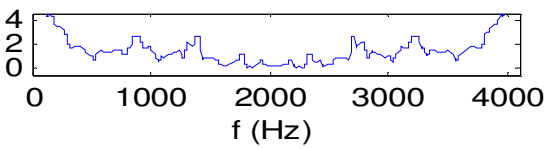

15000 Sample Size

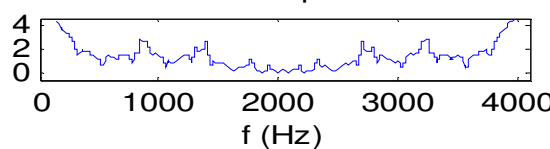

Figure 2: Recovered PSD of Different Sample Sizes Using FFT.
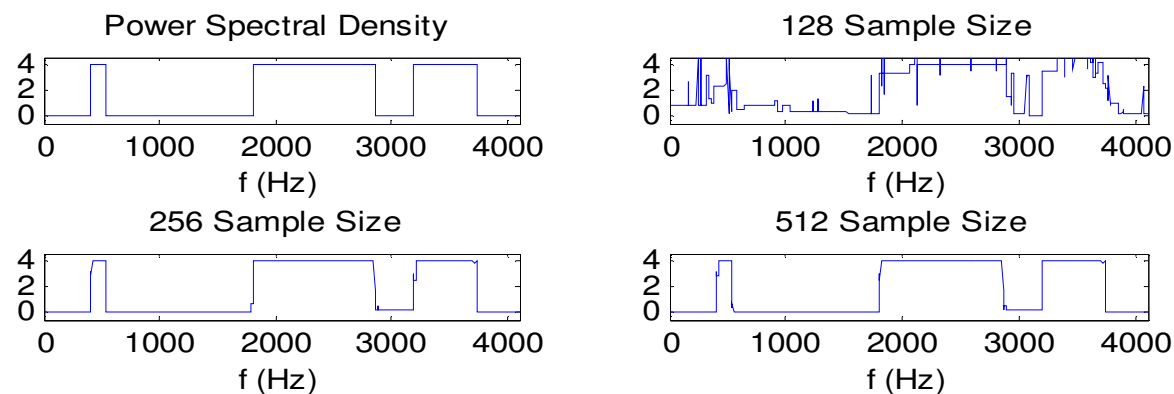

512 Sample Size

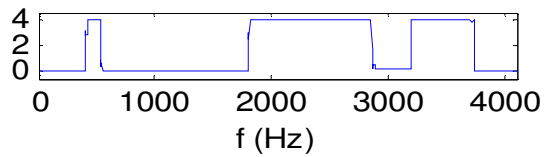

1024 Sample Size

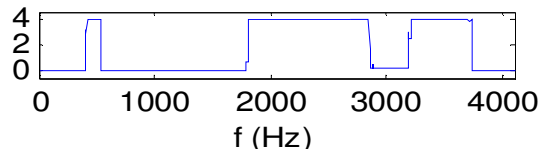

2048 Sample Size

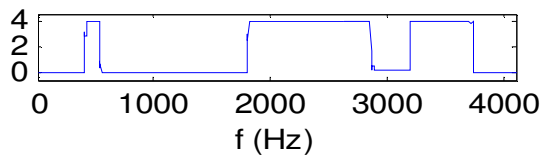

4096 Sample Size

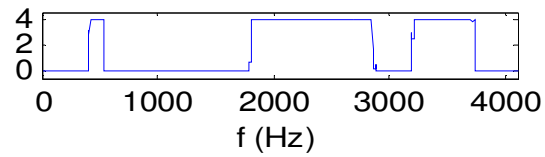

8192 Sample Size

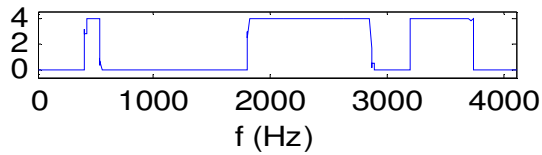

Figure 3: Recovered PSD of different sample sizes using Haar Wavelet.

The reconstructed PSD is taken from an original signal length of 4096. How much sample size is required to reconstruct the PSD is investigated, to show the advantage of CS over traditional methods of sparse signals. The simulation is done using Fast Fourier Transform (FFT) and Haar wavelet basis as a sparsifying basis. Orthogonal Matching Pursuit reconstruction algorithm is used to reconstruct the PSD. Using FFT significantly a high sample size above 15000 is needed to be able to reconstruct the PSD through the CS algorithm. The result obtained for FFT as a basis function is shown in Fig. 2. On the other hand as shown in Fig. 3, using Haar wavelength 256 of sample sizes is enough to reconstruct the PSD. Power Spectral Density of different 
spectrum occupancy is used to show the performance of the two bases considered. Only two of such results were presented in this paper.

We also try to consider the effect of different reconstruction algorithms on the performance of the detection. First we consider a noiseless condition and investigated reconstruction at different sampling rates using the earlier mentioned reconstruction algorithms. At a sampling ratio of $70 \%$, the result obtained from the three reconstruction algorithms that were considered is shown in Fig. 4.
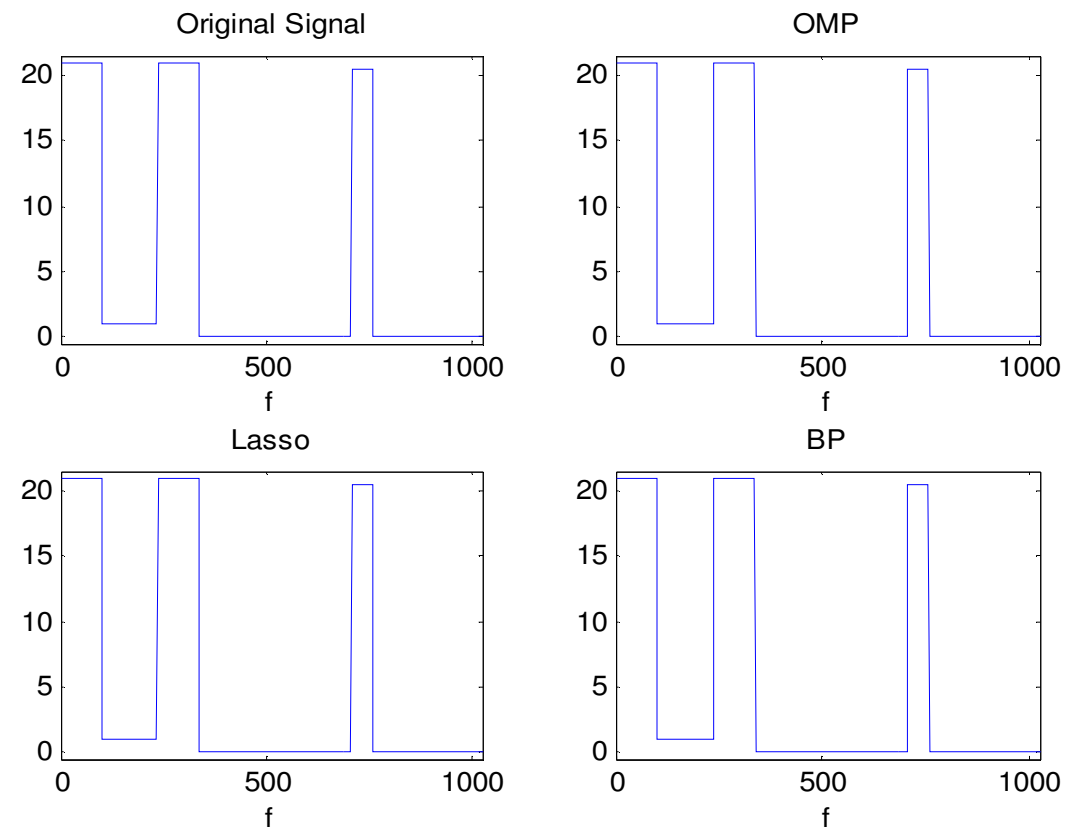

Figure 4: Noiseless Signal frequency response at a compression ratio of $70 \%$

We also investigated the effect of an AWGN channel condition on the detection through CS reconstruction and the result obtained for a situation whereby the signal to noise ratio is $10 \mathrm{~dB}$ is shown in Fig. 5.
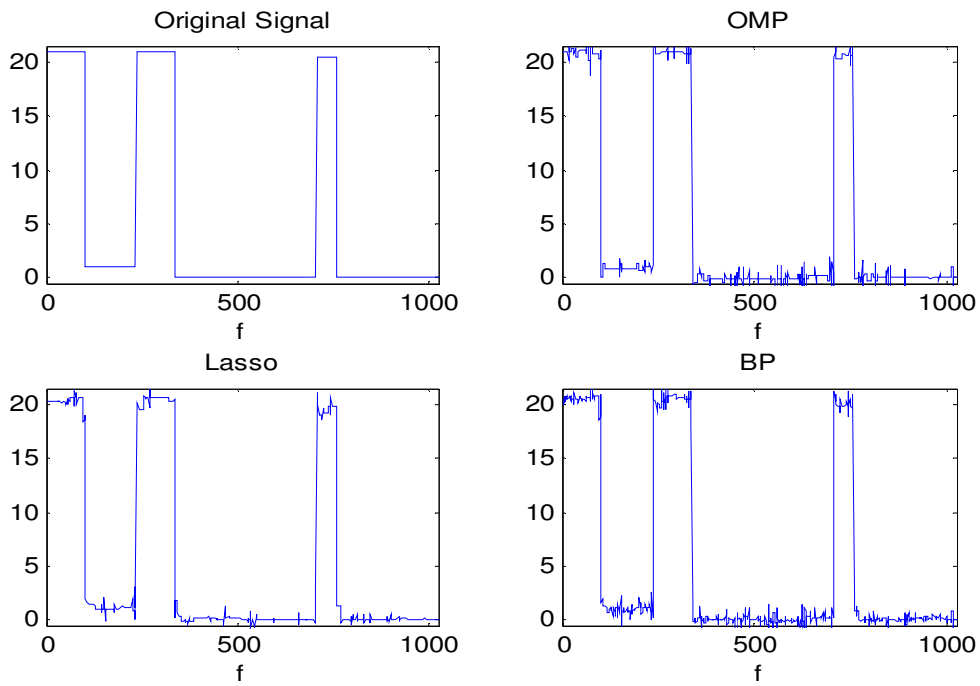

Figure 5: Noisy Signal (10 dB) frequency response at a compression ratio of $70 \%$ 


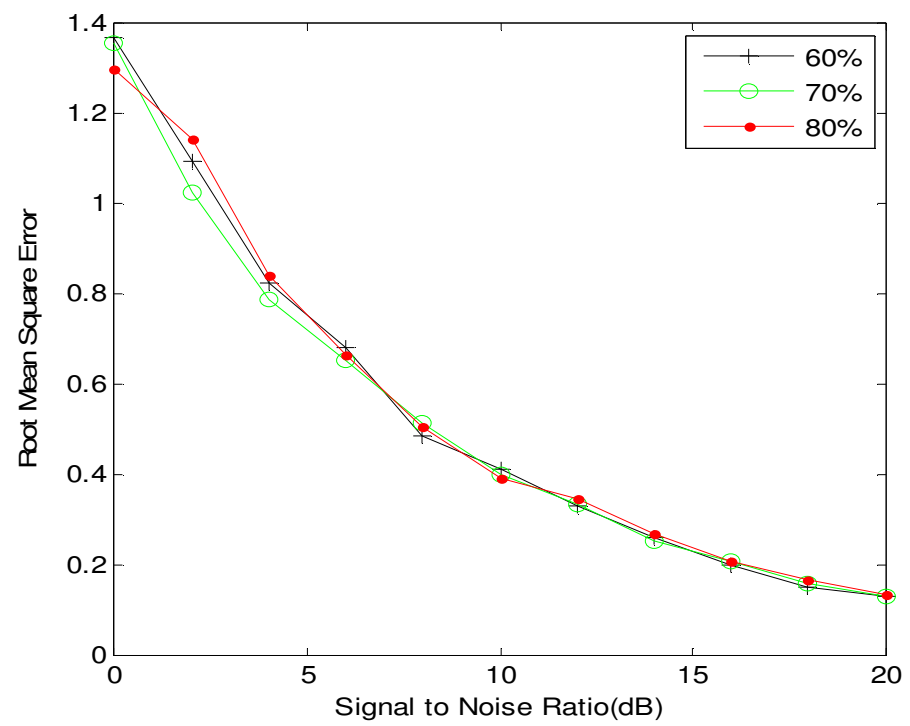

Figure 6: Root Mean Square error using BP algorithm and different no. of samples for AWGN channel

Fig. 6 shows the characterization result obtained using Basis Pursuit algorithm under different signal to noise ratio for an additive white Gaussian noise channel condition. The result was obtained after over 100 iterations. It is a plot of the root mean square error between the reconstructed spectrum and the original spectrum at different noise level for different sampling rates.

We also decided to investigate the behavior of the system in the presence of multiplicative noise. We carried out a test by passing the signal through Rayleigh fading channel in addition to additive white Gaussian noise channel. The reconstructed signal is as shown in Fig. 7 and the characterization result of the root mean square error, using Basis Pursuit algorithm at different sampling rates was shown in Fig. 8.
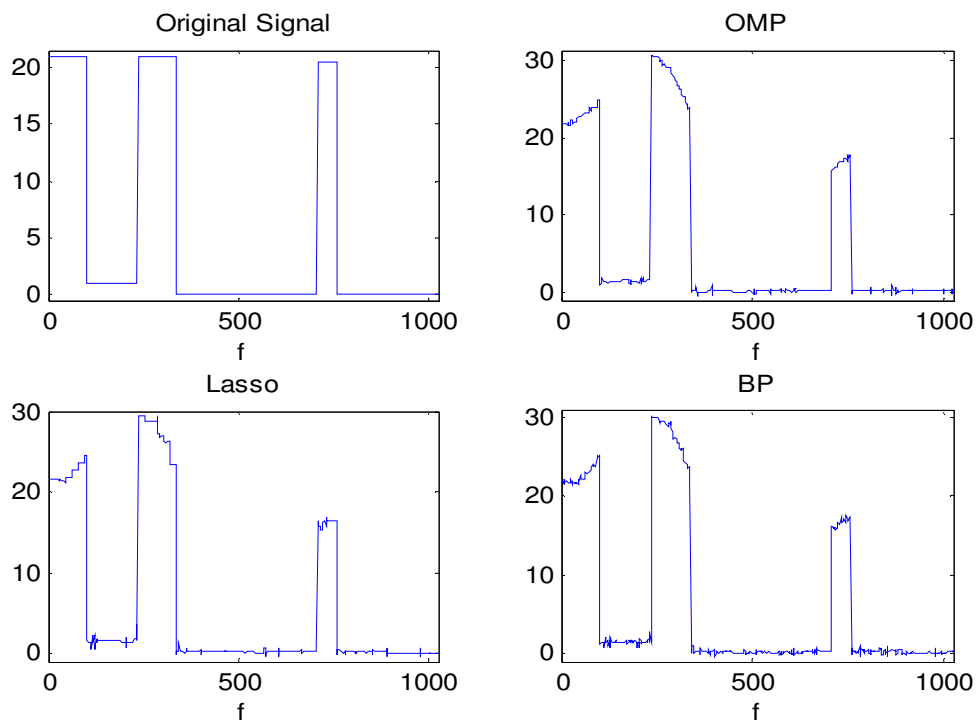

Figure 7: Noisy Signal (Rayleigh fading channel + Gaussian) frequency response at a compression ratio of $70 \%$. 
International Journal of Computer Networks \& Communications (IJCNC) Vol.4, No.2, March 2012

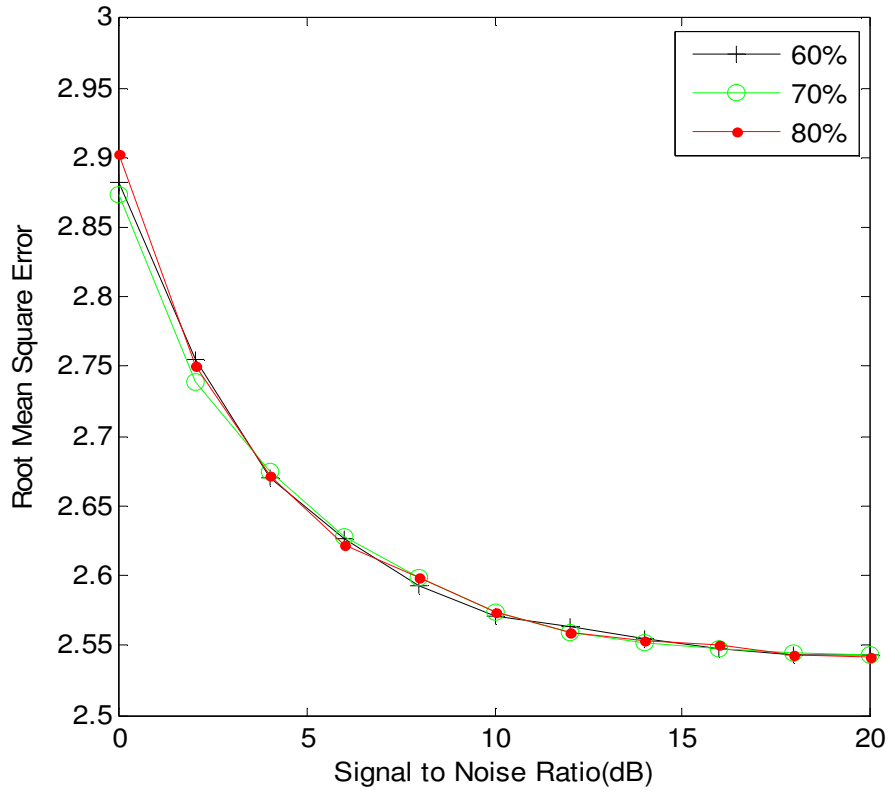

Figure 8: Root Mean Square error using BP algorithm and different no. of samples for Rayleigh Fading + AWGN channel

The behavior of the system under Rice fading channel in combination with AWGN channel was also investigated. Similar results were obtained and they are shown in Fig. 9.
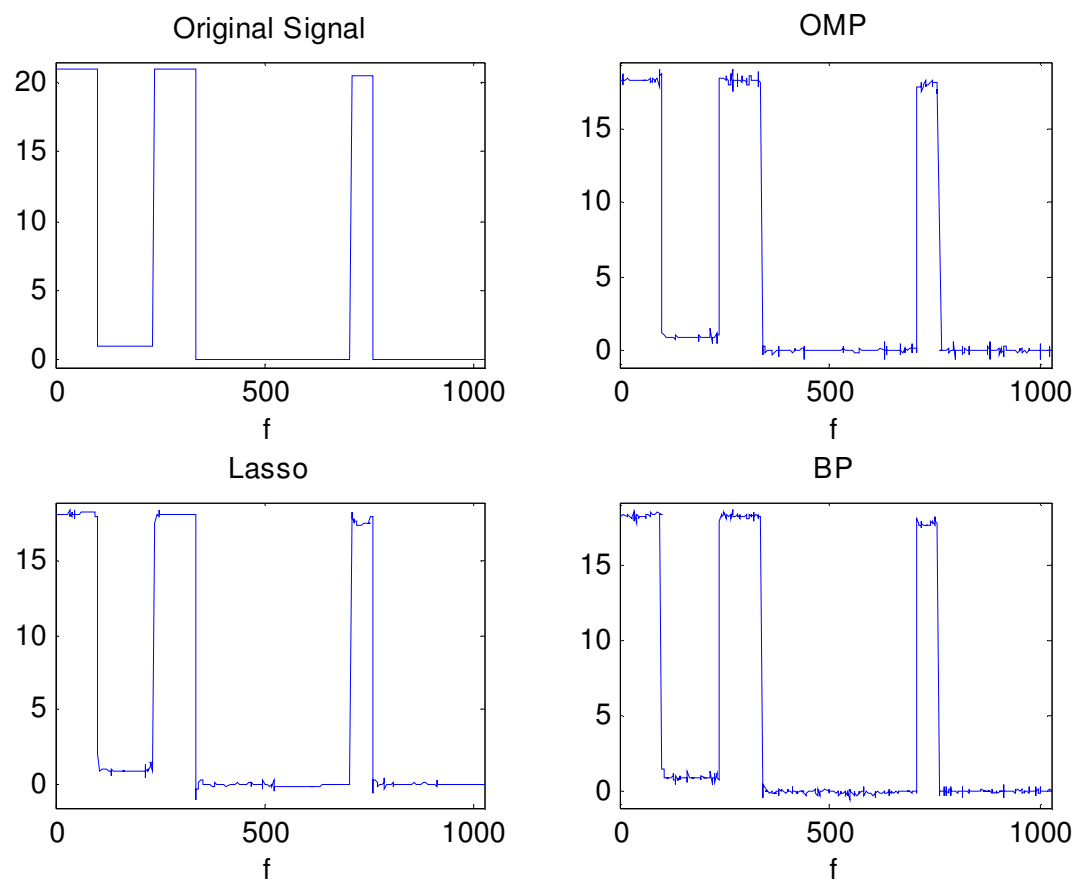

Figure 9: Noisy Signal (Rice fading channel + Gaussian) frequency response at a compression ratio of $70 \%$. 
International Journal of Computer Networks \& Communications (IJCNC) Vol.4, No.2, March 2012

The different algorithms that were considered all performed averagely well in comparison to each other, they are all capable of effectively detecting the occupied bandwidth through CS based signal reconstruction method.
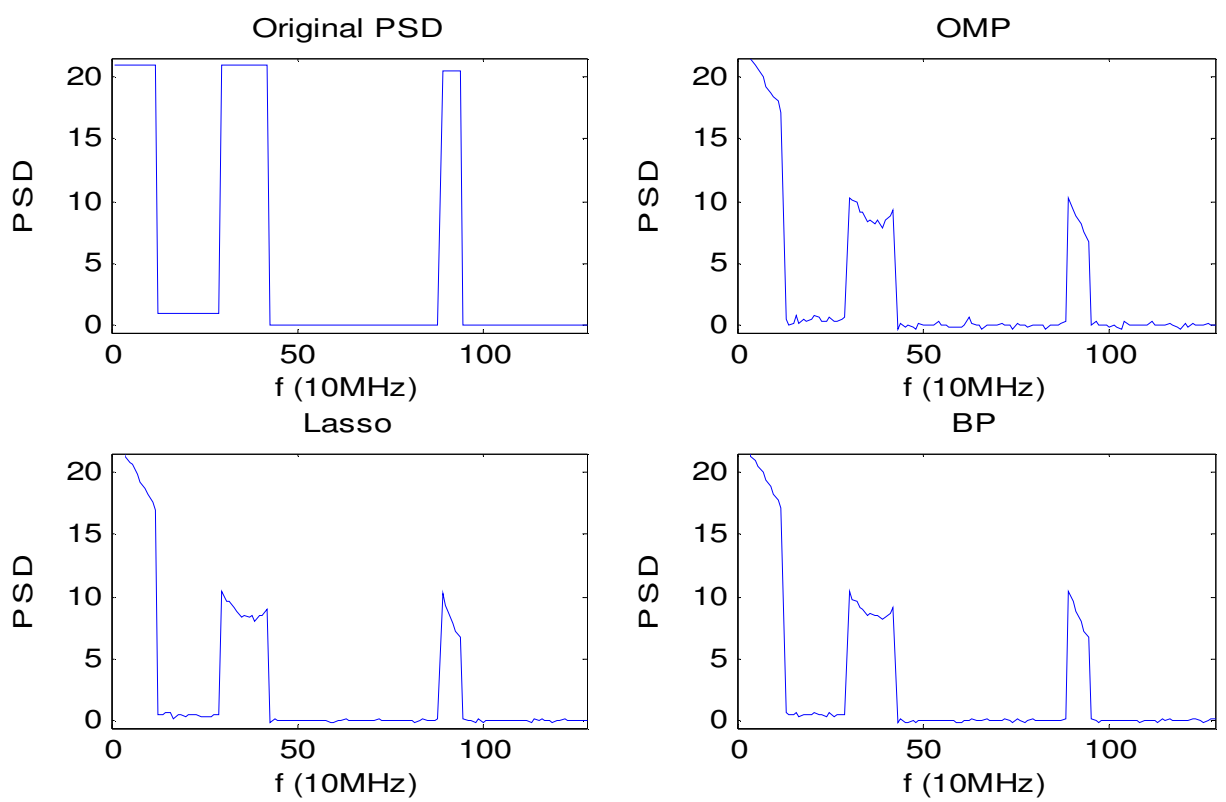

Figure 10: Frequency Reponses PSD Passed Through Fading Channel at 150 Max. Doppler Shift
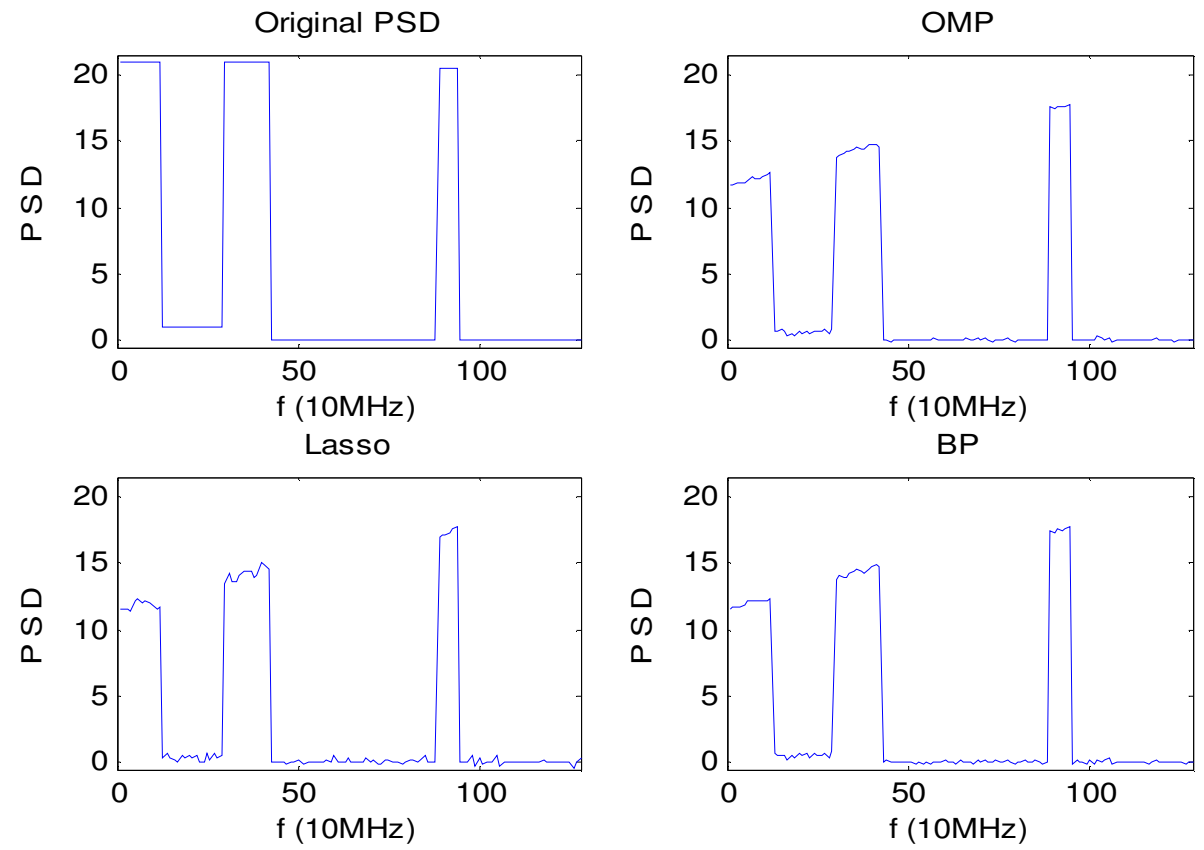

Figure 11: Frequency Response of PSD Passed Through Fading Channel at 100 Max. Doppler Shift 
International Journal of Computer Networks \& Communications (IJCNC) Vol.4, No.2, March 2012
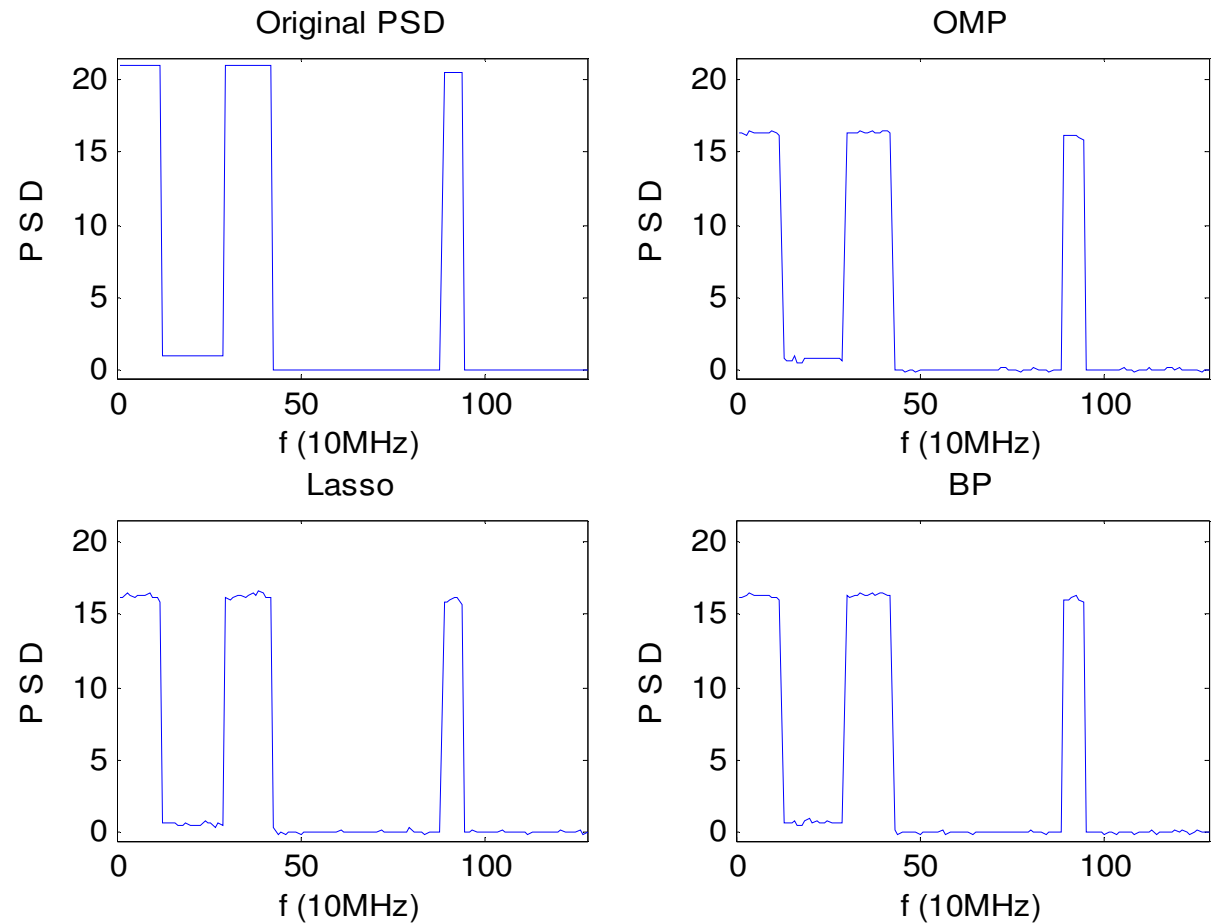

Figure 12 Frequency Response of PSD Passed Through Fading Channel at 10 Max. Doppler Shift

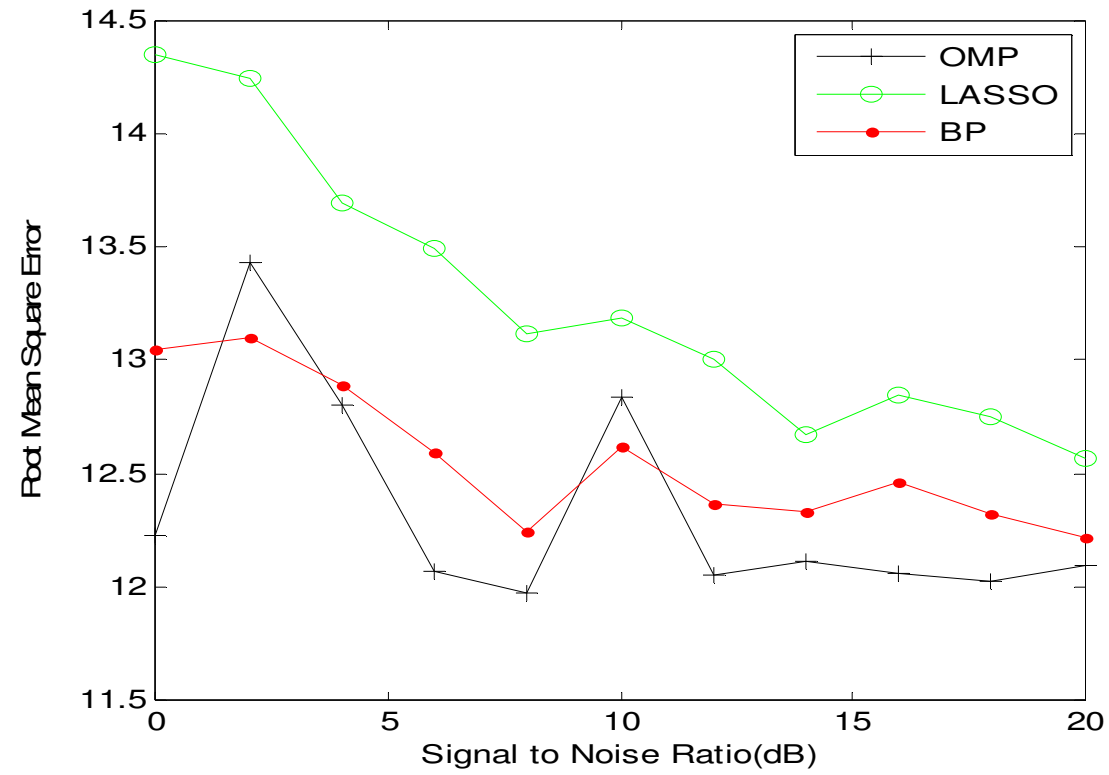

Figure 13: Root Mean Square Error of CS Algorithms in (fading + AWGN) Environment at 150 Max. Doppler Shift 
International Journal of Computer Networks \& Communications (IJCNC) Vol.4, No.2, March 2012

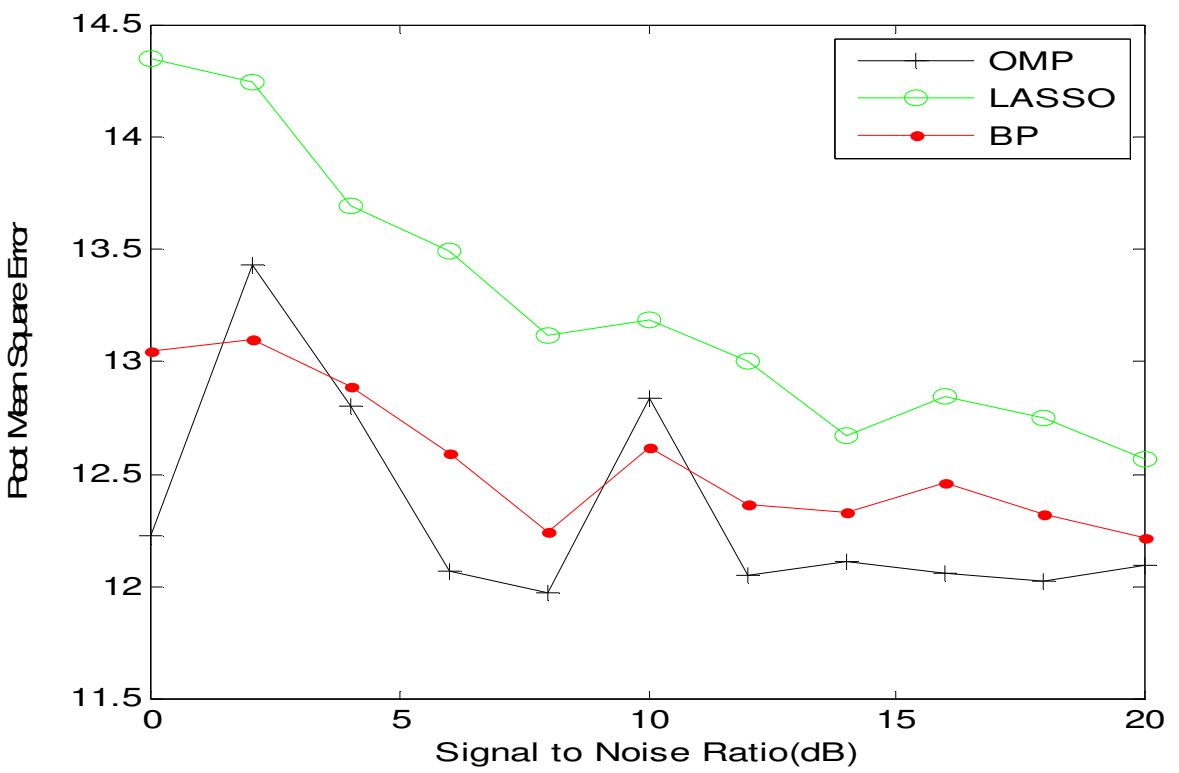

Figure 14 Root Mean Square Error of CS Algorithms in (fading + AWGN) Environment at 100 Max. Doppler Shift

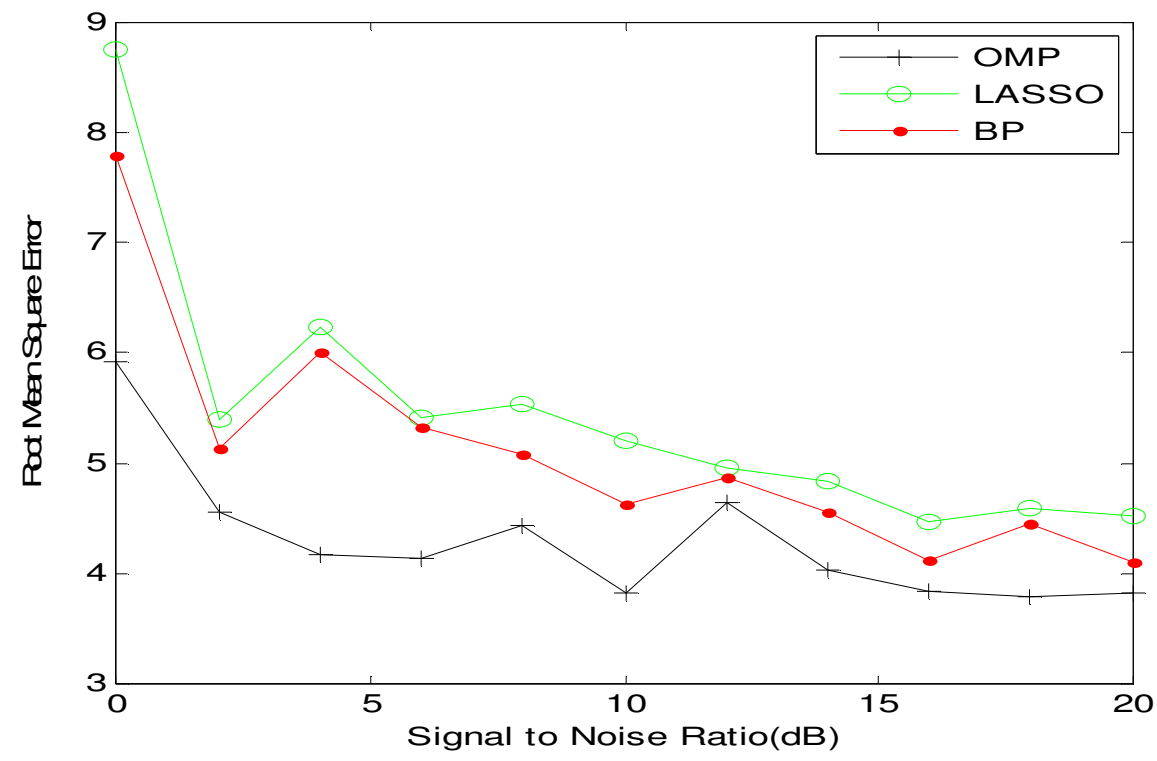

Figure 15 Root Mean Square Error of CS Algorithms in (Fading + AWGN) Environment at 10 Max. Doppler Shift

Figures 10, 11, and 12 consider the effect of Doppler shift on the system performance at different maximum Doppler shifts. As the Doppler shift increases, the channel varies more rapidly, and thus the system performance becomes worse. One can also see that in the low SNR region, the system performance changes only slightly when using the sinc interpolator which is not related to channel state parameters. The results obtained shows that though LASSO perform worst at 150 and 100 Maximum Doppler shift, the result at 10 maximum Doppler shift for all the three algorithms is relatively close. 
However, in the high SNR regime (SNR $>10 \mathrm{~dB}$ ) as shown in Figures 13, 14, and 15, the performance changes significantly. The reason for this is for small SNR values, the AWGN is the dominant factor affecting the accuracy of channel estimation. However, in the high SNR regime, mismatch of the sinc interpolator with the channel variations become the dominant cause limiting the system performance.

\section{Conclusions}

It has been shown that it is possible to determine the available spectrum bandwidth using compressive sampling based signal reconstruction methods. This approach is robust whether in an additive white Gaussian noisy channel or a fading (Rayleigh or Rice) channel. Although significantly error occurs in the magnitude of the characterization based on fading channel plus AWGN in comparison to just AWGN channel, this is not necessarily a problem since the goal is to determine the occupied/unoccupied frequency band in the spectrum. Edge detection algorithms can be employed in determining the unoccupied frequency bands. The effects of Doppler shift on the performance of the CS algorithms at different maximum Doppler shift are considered. It was found that as the Doppler shift increases, the channel fading characteristic varies more rapidly, and thus system performance becomes worse. Also, the three algorithms used performed well at 10 maximum Doppler shift with OMP having the best performance.

The results obtained in this paper show that the proposed CS reconstruction based dynamic spectrum detection method promises to alleviate the current spectrum scarcity problem by using CR networks to opportunistically re-use spectrum holes and enable a lower complexity cognitive radio receiver implementation for wideband spectrum sensing by considerably reducing the number of samples required for power spectral density reconstruction.

\section{REFERENCES}

[1] FCC., "White Spaces," ET Docket No. 04-186. Second report and order, (2008).

[2] I. F. Akyildiz, W.-Y. Lee, M.C. Vuran, S. Mohanty, "Next generation/dynamic spectrum access/cognitive radio wireless networks: a survey," Elsevier Comp. Netw. J. 50(13), 2127-2159 (2006)

[3] A. Sahai, N. Hoven, and R. Tandra, "Some fundamental limits on cognitive radio," Proc. Allerton Conf. Monticello, (2004).

[4] S. Shankar, N. C. Cordeiro and K Challapali, "Spectrum agile radios: Utilization and sensing architectures," Proc. 1st IEEE Symposium New Frontiers Dynamic Spectrum Access Network (DySPAN)Baltimore MD, 160-169(2005).

[5] Rajagopal Sreenivasan, Sasirekha GVK and Jyotsna Bapat, " Adaptive Cooperative Spectrum Sensing Using Group Intelligence," International Journal of Computer Networks and Communication (IJCNC), Vol. 3(3), (2011).

[6] N. Han, S. Shon, J. H.Chung and J. M. Kim, "Spectral correlation based signal detection method for spectrum sensing in IEEE 802.22 WRAN systems," Proc. Int. Conf. Adv. Commun. Technology Phoenix Park Korea, 1765-1770(2006).

[7] G. Wornell, "Emerging applications of multirate signal processing and wavelets in digital communications," Proc. IEEE, Vol. 84, Issue 4 (1996).

[8] Z. Tian and G. B. Giannakis, "A wavelet approach to wideband spectrum sensing for cognitive radios," Proc. 1st Int. Conf. Cognitive Radio Oriented Wireless Network Communication (CROWNCOM) Greece, 1-5(2006).

[9] Y. Hur, et. al., "A cognitive radio (CR) system employing a dual-stage spectrum sensing technique: A multi-resolution spectrum sensing (MRSS) and a temporal signature detection (TSD) technique," Proc. IEEE Global Telecommunication conference San Francisco CA, 40904093 (2006).

[10] Emmanuel Candes, and Terence Tao, "Decoding by Linear Programming," IEEE Transactions on Information Theory, 51(12), (2005).

[11] Jarvis Haupt and Robert Nowak, "Compressive Sampling for Signal Detection," Proc. IEEE Int. Conf. on Acoustics Speech and Signal Processing (ICASSP), (2007). 
International Journal of Computer Networks \& Communications (IJCNC) Vol.4, No.2, March 2012

[12] Cajetan M. Akujuobi, Olusegun Odejide and Jerry Fudge, "Development of Wavelet Based Signal Detection and Measurement Algorithm," Proc. Of ASEE 6th Global Colloquium on Engr. Edu. Istanbul Turkey, (2007).

[13] Z. Tian and G. B. Giannakis, “Compressed Sensing for Wideband Cognitive Radio,” Proc. IEEE Int. Conf. on Acoustics Speech and Signal Processing (ICASSP), 1357-1360 (2007).

[14] Y.Liu and QQ. Wan, "Compressive wideband spectrum sensing for fixed frequency spectrum allocation." arXiv:1005.1804, 2010.

[15] Farrag, M.; El-Khamy, M.; El-Sharkawy, M.; , "Optimized bases compressive spectrum sensing for wideband cognitive radio," Personal Indoor and Mobile Radio Communications (PIMRC), 2011 IEEE 22nd International Symposium on, vol., no., pp.305-309, 11-14 Sept. 2011

[16] Hong, S.; , "Multi-Resolution Bayesian Compressive Sensing for Cognitive Radio Primary User Detection," Global Telecommunications Conference (GLOBECOM 2010), 2010 IEEE , vol., no., pp.1-6, 6-10 Dec. 2010

[17] W.C.Y. Lee, "Mobile Communications Engineering: Theory and Applications." Second Edition, McGraw-Hill Incorporated, New York, April 1998.

[18] W. C. Y. Lee, "Mobile Communications Design fundamentals", Second Edition, Wiley - InterScience, January 1993.

\section{Authors}

Olusegun O. Odejide received the B.S. degree in electrical and electronic engineering from University of Ibadan, Ibadan, Nigeria in 1997. He received the M.S. degree in Computer and Information Systems Engineering from Tennessee State University in 2004 and Ph.D degree in Electrical Engineering from Prairie View A\&M University, Prairie View, Texas in 2009. He recently received the prestigious NRC research associateship fellowship. His research interests are in the areas of Signal/Image/Video Processing, compressive sensing and Communication Systems.

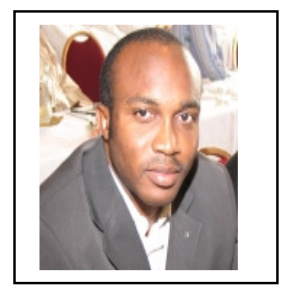

Michael O. Odeyomi received the B.S. degree in electronic and computer engineering from Lagos state University, Nigeria in 2005. He received the M.S. degree in Electrical Engineering from Prairie View A\&M University, Prairie View, Texas in 2010. He currently works for Shell as a Business Infrastructure Management Consultant. His research interests are in the areas of Signal Processing and Communication Systems.

Annamalai A. Annamalai is presently the Director of Center of Excellence for Communication Systems Technology Research; a Texas A\&M Board of Regents approved University Research Center at the Prairie View A\&M University, and a tenured faculty member in the Department of Electrical and Computer Engineering. He has over 16 years of research/teaching experience in wireless communications at Motorola, University of Victoria, Air Force Research Laboratory, Virginia Tech and PVAMU with approximately 200 peer-reviewed publications and 5 book chapters. Dr. Annamalai has been honored by his

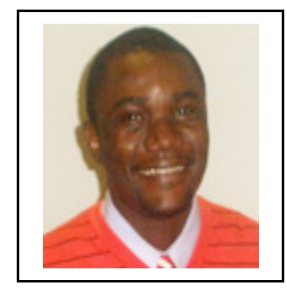
colleagues on numerous occasions for his excellence in research including winning the 2011 Roy G. Perry College of Engineering Outstanding Faculty (Research) Award, IEEE Leon Kirchmayer Prize Paper award, ASEE/AFOSR Summer Faculty Fellowships, NSERC Doctoral Prize, CAGS/UMI Distinguished Doctoral Dissertation Award, IEEE VTS/Motorola Daniel E. Noble Fellowship, among others. He had served on the Editorial Boards of four IEEE journals/transactions in the last 12 years, and has helped to organize a few major IEEE conferences on wireless communications including serving in the capacity of Technical Program Chair of the 2002 IEEE Vehicular Technology Conference in Vancouver, Canada. His current research interests include cooperative spectrum sensing, compressive sensing, cross-layer design for scalable multimedia transmission and cooperative wireless communications. 